\title{
Present Situation of Radioactivity
}

Distribution

\author{
-Summary of Initial Activity Situation-

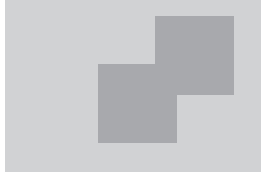 \\ Tohoku University, Takashi Nakamura \\ Japan Atomic Energy Agency, Takuma Momose \\ Nagoya University, Tetsuo Iguchi
}

\begin{abstract}
This paper outlines the issues discovered during the initial activities related to the monitoring of radioactive contamination during the Fukushima Daiichi Nuclear Power Plant Accident, individual exposure dose assessment, the radiation measurement in the environment by general public, as well as their future prospects.
\end{abstract}

\section{Radioactivity Distribution}

After the Fukushima Daiichi Nuclear Power Plant Accident, airborne surveys, radiation dose measurements, and sampling/analysis of soil were conducted to understand the contamination level of the soil. The items that were measured, analyzed, and assessed have been published in sequence by the Ministry of Education, Culture, Sports, Science and Technology (MEXT). As a part of this activity, an examination committee for producing the radiation dose distribution map was established at the MEXT in late May 2011. This committee held 10 meetings by September 30, 2011. They worked with over 400 collaborators from more than 90 organizations, including universities and research institutes, to conduct the following measurements twice from June 6 to June 13 and from June 27 to July 8.

(1) Conduct a survey of environmental dose rate using a vehicle called KURAMA developed by Kyoto University.

(2) Select a spot at every $2 \mathrm{~km}$ within $80 \mathrm{~km}$ radius of Fukushima Daiichi Nuclear Power Plant and at every $10 \mathrm{~km}$ within $80-100 \mathrm{~km}$ radius and the rest of Fukushima Prefecture. On each spot, collect five soil samples from a depth of $5 \mathrm{~cm}$ within the $3 \mathrm{~m} \times 3 \mathrm{~m}$ area. Measure the radioactive concentration by the nuclide of each sample using a Ge detector.

(3) Use a scintillation survey meter to measure the environmental dose rates of the spots where soil samples were collected.

(4) Conduct related research on the depth-direction radioactive materials distribution in the soil, study of the migration of radioactive materials accumulated on the soil surface, and the study of radioactive material in the rivers and ground water.

On August 2 and 12, the results of the airborne survey, the vehicle-borne survey, and the

DOI : $10.15669 /$ fukushimainsights.Vol.1.248

(C) 2021 Atomic Energy Society of Japan. All rights reserved.

Originally published in Journal of the Atomic Energy Society of Japan (ISSN 1882-2606), Vol. 54, No. 11, p. 743-746

(2012) in Japanese. (Japanese version accepted: August 17, 2012) 
fixed-point measurement were published first as a distribution map of environmental dose rate. On August 29, the soil concentration distribution maps of Cs-134 and Cs-137 and the result of in situ measurement with Ge detector were published. On September 13, the result of migration of cesium in forests was published. Following these, the soil concentration distribution map of I-131 was published on September 21. On September 30, the soil concentration maps of Sr-89, Sr-90, Pu-238, and Pu 239+240 were published. The distribution maps of other rare radioactive nuclides and the research result of migration to rivers and other waters are scheduled to be published in the future.

Due to limited space, we will only include one example from these published data. Figure 1 shows the soil concentration distribution map of Cs-137 (published on August 30, 2011). These results reveal that both the environmental dose rate measurements and the soil concentration distribution map of Cs-137 show the highest value in the northwestern direction from the nuclear power plant; higher values are observed toward the Nakadori region of Fukushima Prefecture. The general tendency of this distribution corresponds well with the result of the airborne survey. Moreover, it became clear that there is strong correlation between the environmental dose rate of each spot and the soil concentration distribution of Cs- 137 . This distribution map also corresponds well with the result of SPEEDI calculation and clearly shows that the distribution was dominated by the weather condition at that time. Thus, it is a highly valuable data for understanding the current soil contamination situation and devising the future environmental recovery; it is desirable to conduct a continuous monitoring in a wider area.

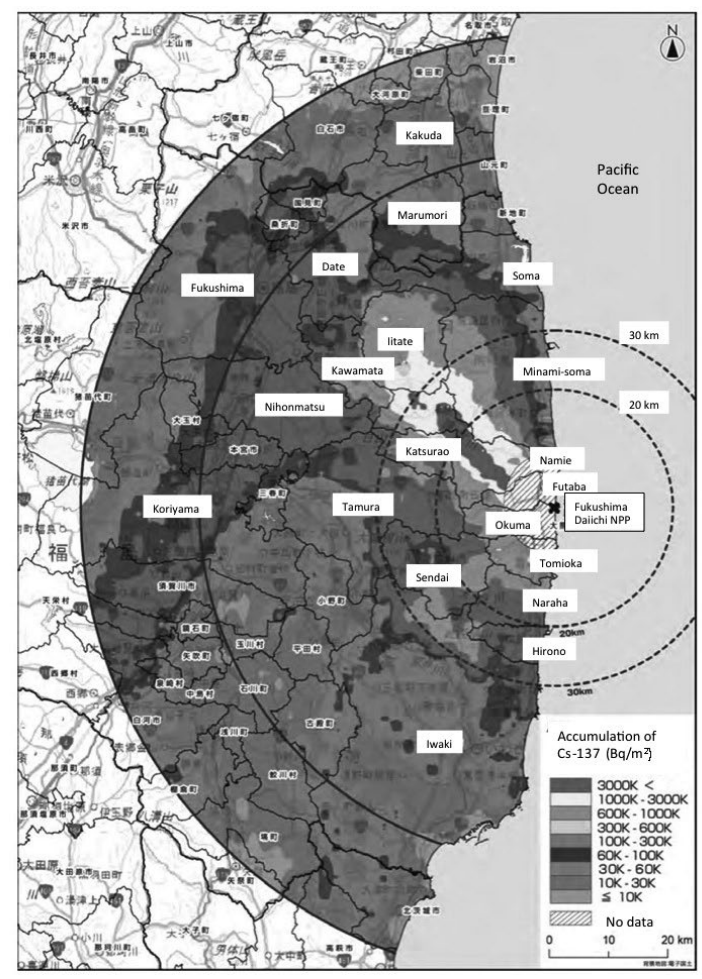

Figure 1 Soil concentration map of Cs-137 (http://radioactivity.mext.go.jp/old/ja/1910/2011/09/1910_0912. pdf (Appendix 4) 


\section{Individual Monitoring of Workers and Residents}

\section{Individual Monitoring of Workers}

The workers in the premises of Fukushima Daiichi Nuclear Power Plant were conducting their emergency work under a severe working environment with radiation during the early stage of the accident. The observed maximum environmental dose rate reached $12 \mathrm{mSv} / \mathrm{h}$ (March 15, 2011), and it became necessary for the workers to wear a mask not only outside but also inside the building for protection against internal exposure. Moreover, female workers who stayed in the important anti-seismic building and other buildings suffered from internal exposure by radioactive iodine and other materials and their exposures exceeded the dose limit for women. In addition, flooding by the tsunami made many individual dosimeters (APD) and its reading system unusable, causing the shortage of individual dosimeters. The wholebody counter (WBC) installed inside the power plant became unusable due to the increase in background and defects. Under such a circumstance, external exposure dose was initially measured using a method wherein each work group shares one dosimeter. After April, the necessary number of individual dosimeters was secured. For measuring internal exposure dose, the onboard WBC of Japan Atomic Energy Agency (JAEA) dispatched to Onahama was used. The workers whose internal exposure rate may have exceeded $20 \mathrm{mSv}$ according to this test underwent a measurement using the precision-type external measurement device with Ge semiconductor detector inside the 20 -cm-thick iron shield installed in the radiation infirmary of the nuclear fuel cycle engineering laboratory of JAEA.

The distribution of the effective dose, which is the sum of the external exposure and internal exposure of the workers engaged in the emergency work, has been published by Tokyo Electric Power Company. According to this, six workers exceeded the dose limit of $250 \mathrm{mSv}$ for an emergency work by late March and the maximum effective dose was $678 \mathrm{mSv}(88 \mathrm{mSv}$ for external exposure and $590 \mathrm{mSv}$ for internal exposure). The main cause of the exposure that exceeded the limit dose was internal exposure by radioactive iodine. The exposure management of the workers was strengthened after April, and there has not been any case wherein the exposure exceeded $50 \mathrm{mSv}$ since May.

\section{Individual Monitoring of Residents}

As a part of health management survey of Fukushima Prefecture, the activity survey of every prefectural resident after the earthquake is ongoing. Based on the result of this survey, external exposure dose assessment of each resident, considering the time course of the environmental dose rate due to $\gamma$ ray, differences in position and the shielding effect of buildings into consideration, will be conducted. Moreover, in some areas, individual dosimeters are given to school children to measure their individual external exposure doses in their living environment.

The residents within the restricted area or the deliberate evacuation area may have been significantly exposed to internal exposure mainly due to inhalation of radioactive materials in plume during the early stage of the accident. If there is a result of continuous monitoring of radioactive material concentration in the atmosphere, the internal exposure situation of the area by calculating the environmental dose rate. However, due to the blackout caused by the earthquake, monitoring data of radioactive material concentration in the atmosphere in the area around the nuclear power plant is scarce. Although there are measurement results of iodine or dust samples conducted around the power plant from March 12 to March 15 by the 
environmental monitoring team dispatched by the national government to the off-site center during the early stage of the accident, these data are not continuously monitored and because they have shortcomings such as the limited measurement points and period, they are difficult to apply to internal exposure dose assessment of the residents.

Examples of continuous monitoring include the continuous dust monitoring conducted form March 13 by the Nuclear Fuel Cycle Engineering Laboratory in Tokai village, Ibaraki Prefecture. Table 1 shows the actual measurement data of the radioactive material concentration data in the air by each nuclide. Based on this result, the internal exposure dose from March 13 to May 23 in the measurement point (outside) was assessed using the inhalation rate of general public. The result showed that the effective dose of an adult was $0.57 \mathrm{mSv}$ and the thyroid equivalent dose of a small child (one year old) was $15 \mathrm{mSv}$.

A study on internal exposure dose by Cs-134 and Cs-137 using WBC was conducted from July to late August by the National Institute of Radiological Sciences and JAEA for about 3,300 residents of Namie, Iitate, and Yamakiya areas of Kawamata. The measurement has continued since September by JAEA and other organizations for residents in seven municipalities of Futaba District. In all the measurements, numerical results that would affect health were not detected. A plan to organize WBC and start measurement in order at other areas of Fukushima Prefecture is being prepared. The result of the tests with WBC is planned to be registered in the health management survey database of Fukushima Prefecture.

Table 1 Concentration of radioactive materials in the atmosphere in Tokai, Ibaraki Prefecture

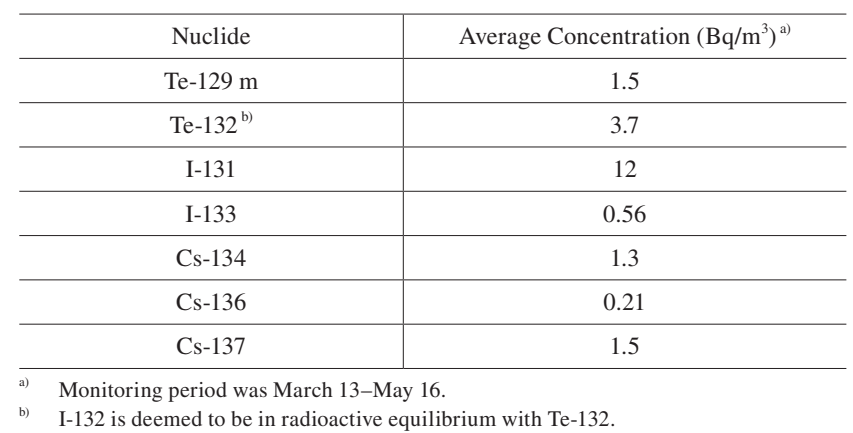

\section{Future Prospect}

For the reconstruction of the dose of residents, it is difficult to assess the internal exposure by materials, such as radioactive iodine, during the early stage of the accident. During late March this year, the pediatric thyroid examination was conducted on 1,080 children from Iwaki and other places. Its result showed that every child exhibited a screening level of thyroid equivalent dose $\leq 100 \mathrm{mSv}$. Thus, the contribution of internal exposure dose, including inhalation during the evacuation, is smaller compared to the external exposure dose from, for instance, the $\gamma$ ray of Cs-137 that deposited on the ground. However, sufficient evidence-based data is not available for proving this prediction. When focusing on the I-131/Cs-137 ratio of deposited dust within $30 \mathrm{~km}$ radius, while it was $0.31-5.83$ in the north to southwest direction, including the area around Iitate where the amount of radioactive material deposition was high, the ratio of I-131 was comparatively large at 13-26.5 in the south to southwest direction, where the amount of radioactive material deposition was relatively low. As can be seen in this case, the fact that I-131/Cs-137 ratio can be different depending on the direction or time also needs to be considered. When the detail of the concentration distribution on radioactive 
material in the atmosphere becomes clear in future via comparison with the vast environmental monitoring data and the calculation result of atmospheric distribution simulation of radioactive material using, for instance, SPEEDI, it is expected that the actual measurement of Cs-137 and other materials by WBC will be used as an individual index for assessing the internal exposure dose of inhalation of nuclides such as radioactive iodine.

\section{Points of Caution for Radiation Measurement}

\section{Introduction}

As the extent of release of large quantity of radioactive materials into the environment and their diffusion into a large area after the Fukushima Daiichi Nuclear Power Plant Accident became clearer, interests and concerns for radiation around us, for instance, in our living environment, food, drinks and everyday goods, are increasing. Currently, the MEXT and the Ministry of the Environment are leading the governmental initiative to gain a detailed understanding of radioactive contamination based on actual measurements. Meanwhile, people who are not specialized in radiation measurement are willing to post their measurements on websites and blogs or identify the spots with high dose rate and share the information with each other to actively take precautionary measures. To correctly understand these radiations around oneself as seen in these example (including the natural radiation that existed before the accident) will lead to in-depth understanding of various radiation risks and measures to reduce them. Therefore, we believe that the Atomic Energy Society of Japan should aggressively promote and support these movements.

However, measurement of radiation, particularly quantitative evaluation of exposure dose to human body or the radioactive concentration in articles, is one of the most difficult techniques among the measurement of various physical quantities (e.g. time, length, and weight). A variety of miscellaneous radiation measuring instruments are available on the market. However, to make effective use of the activities of radiation measurement of daily activities by general public, it is strongly desirable that they understand the characteristics specific to each measuring instrument and measured values, and the characteristics of radiation in the environment and target actually being measured.

Here, we will focus on the measurements of "environmental dose rate," "surface contamination of human body/articles," and "radioactivity concentration in food/drink" that are with high concern among the public. The discussion will be conducted from the following perspectives: (1) What does the instrument measure? (2) How to convert the measured data to dose rates or radioactivity concentrations. (3) To what extent do the measured results vary? From these three perspectives, we will present an overview of the points of consideration in the radiation measurement for the general public.

\section{What does the Instrument Measure?}

Almost all current radioactive materials in the environment originated from the Fukushima Daiichi Nuclear Power Plant Accident can be radioactive Cs (Cs-134 and Cs-137). From these radioactive Cs, $\gamma$ ray (photon) and $\beta$ ray (electron) are emitted. When they collide with materials, electric charge or light is produced. Radiation measuring instruments use gas, such as air or noble gases, or solid matter, such as transparent inorganic fluorescent materials or semiconductors, as sensing materials. For instance, common small radiation survey meter 
types often used for relatively low environmental dose rate in the surrounding area outside Fukushima Prefecture include Geiger-Mueller (GM) counter tube of the former type and scintillation detector of the latter type.

In the GM counter tube type, the electric charge generated in the filled gas per one radioactive substance that entered the counting tube is amplified by the discharge phenomenon to generate a pulse current (or voltage) of a substantially constant magnitude. Thus, by counting the number of current pulses, the intensity of the radiation can be measured. Meanwhile, the scintillation type normally combines material that generates fluorescence when hit by radiation, such as $\mathrm{NaI}(\mathrm{Tl})$ or $\mathrm{CsI}(\mathrm{Tl})$ crystals (scintillator), with photomultiplier tube or photodiode that converts light to electric current. As the intensity of fluorescence light or the magnitude of current pulse is almost proportional to the radiation energy absorbed by the scintillator, energy information can be measured together with the strength of radiation. By multiplying the electric signals obtained from these radiation measuring instruments with conversion factors based on calibration experiment or theoretical calculation, the radioactivity expressed in units of Becquerel (Bq) (the number of radioactive substance released per second) or Sievert ( $\mathrm{Sv}$ ) (equivalent or effective) dose (in the case of $\gamma$ rays and $\beta$ rays, equivalent to the amount of energy absorbed per $\mathrm{kg}$ of human body tissue) are obtained.

\section{Correct Measurement Method with Radiation Survey Meter}

First, the small radiation survey meters available on the market are electronic product that can be easily influenced by the surrounding although they are generally robust and easy to use. Thus, it is the most basic precondition to follow the instructions on the temperature, humidity, air pressure, vibration, shock, and electromagnetic induction, during measurement written in the manual. Moreover, it is extremely important to use a meter with an understanding of its basic performance as a radiation meter to achieve a correct interpretation of the measurement results. Here, we will discuss the main points of caution of small radiation survey meters available on the market from the perspective of detection sensitivity, energy dependency, and directional dependency.

\section{(1) Detection Sensitivity}

The minimum detection limit of hourly environmental dose rate with a small radiation survey meter available on the market is $\sim 0.1 \mu \mathrm{Sv} / \mathrm{h}$ with the GM counter type and $\sim 0.01 \mu \mathrm{Sv} / \mathrm{h}$ with the scintillation. Thus, it is desirable to choose the scintillation type to correctly measure the low dose rate in a normal environment. However, as discussed in section III-2, a high-precision standard radiation source and calibration under a strict measurement condition are required to convert the pulse counting rate (count/second) of the electric signal from the radiation measuring instrument outputs into dose rate $(\mu \mathrm{Sv} / \mathrm{h})$. A correct dose rate can only be obtained when the measurement is conducted under the same condition as that of the calibration. Moreover, the pulse counting rate that is equivalent to the minimum measurement limit is in the order of $\sim 0.1 \mathrm{cps}$. Unless a sufficient measurement time is given, the measurement result essentially exhibits variation. Considering this minimum measurement limit, it is extremely difficult to measure the weak radioactivity that is equivalent to the radioactive Cs in the regulatory limit $(\sim 100 \mathrm{~Bq} / \mathrm{kg})$ contained in food with a common radiation survey meter.

\section{(2) Energy Dependency}

Although to measure dose rate accurately, it is desirable for the energy dependency (detector response) of the detection efficiency to be equivalent to the energy dependency of $1 \mathrm{~cm}$ 
dose equivalent (exposure dose under $1 \mathrm{~cm}$ of skin), there is often a deviation. Among scintillation-type survey meters that can measure radiation energy, there is a model (energy-compensation type) that compensates the deviation by weighting the energy discrimination measurement. However, for the relatively inexpensive and small GM counter-type and scintillation-type survey meter, energy compensation is not performed, even though they devise means for compensation such as physically attaching a filter (cover). Thus, they have a tendency to improve the detection efficiency of $\gamma$ ray whose Cs-137 $\gamma$ ray energy is $\sim \leq 0.66 \mathrm{MeV}$. This means that even if it is calibrated by a Cs-137 $\gamma$ ray source, it can easily show higher measurement values when low energy $\gamma$ ray, which are scatter components, exist in a large amount in the environment.

\section{(3) Directional Dependency}

The directional dependency of the detection efficiency of radiation survey meter depends on the shape of the sensitivity area. Because the GM counter type is rod shaped, its detection efficiency is low at front and back and high on the sides. Moreover, the directional characteristic of the scintillation type is relatively isotropic. However, the detection efficiency of the direction where photoelectric conversion device is located tends to be lowered by $\sim 20 \%$. Thus, to conduct dose measurement accurately, it is necessary to always measure from the same direction and facing the same side of the measurement object as the calibration.

\section{(4) Statistical Properties of Counts from Radiation Measuring Instrument}

The counts from radiation measuring instrument have an essentially fluctuating property (the variation of the counts follow Poisson distribution or normal distribution). When the count value is $N$ counts, its fluctuation index, standard deviation $\sigma$, is known to be $\sqrt{N}$. Thus, the lower the sensitivity of the meter or the lower the dose being measured, the greater the fluctuation becomes. For instance, with a small GM counter-type survey meter available on the market, the detection sensitivity is $\sim 10$ counts per minute measurement for $\sim 0.1 \mu \mathrm{Sv} / \mathrm{h}$ environmental dose rate. Therefore, the fluctuation of the relative standard deviation $\sim \pm 30 \%$ $(10 \pm \sqrt{10}$ count) is expected. Note that the "Radioactive concentration measurement methods guideline" by the Ministry of Environment encourages to use three times the standard deviation of the average of multiple dose measurements as the index for the fluctuation.

\section{(5) Other Points of Caution}

When measuring the radiation dose of environment or goods with a small radiation survey meter available on the market, it is important to consider the presence of time variation of naturally existing radiation originating from the location or weather in addition to the indicated value originating from the characteristics of the meter itself. Among the natural radiation, the radiation coming from space has small influence on a small radiation survey meter because it has high energy and is difficult to detect. Meanwhile, for instance, $30 \mathrm{~Bq}$ of K-40 (half-life 1.25 billion years), which is an isotope of potassium (a necessary element for human body), is contained in $1 \mathrm{~g}$ of natural potassium, and it is widely distributed in soil or food. Moreover, if there is granite or other such material, the contribution of dose of thorium, uranium, or radioactive nuclide of their group (for instance, radioactive gas $\mathrm{Rn}-222$ released in the atmosphere and its daughter nuclides) contained in it cannot be ignored. Indeed in Japan, maximum 30\% fluctuation range due to the location or weather of natural radiation for about $\sim 0.1 \mu \mathrm{Sv} / \mathrm{h}$ environmental dose rate is expected.

Moreover, "time constant" is used as the standard for the response time of the meter in a radiation survey meter. Generally, unless the indicated value of dose rate is read after a time period of a few times the time constant has elapsed, underestimation or some deviation in the 
detection location of hotspot would occur.

In addition, when measuring the radioactive Cs of soil surface or goods surface using, for instance, a GM counter-type survey meter that possess sensitivity for both $\gamma$ ray and $\beta$ ray, because the sensitivity for $\beta$ ray is nearly two orders of magnitude higher than for $\gamma$ ray, a wrong dose rate value that is extremely high will be indicated unless the measurement is conducted shielding $\beta$ ray.

\section{General References}

1) Prime Minister's official residence. On the monitoring data of radiation. http://www.kantei.go.jp/ saigai/monitoring/index.html. [in Japanese]

2) JAEA-Review 2011-035. Special environmental radiation monitoring result related to the Fukushima Daiichi nuclear power station accident. [in Japanese]

3) Ministry of the Environment. Guideline relevant to waste material The fifth part Guidelines for method of measurement of radioactive concentration. 2011 December. http://www.env.go.jp/jishin/rmp/ attach/haikibutsu-gl 05_ver1.pdf (This link was not found as of July 26, 2017). [in Japanese] 